\title{
PREPARAÇÃO E AVALIAÇÃO DA PENEIRA MOLECULAR SBA- 15 VISANDO SEU USO NA SEPARAÇÃO ÓLEO/ÁGUA
}

\author{
J. J. RODRIGUES, R. S. EDUARDO, E. L. DA SILVA e M. G. F. RODRIGUES \\ Universidade Federal de Campina Grande, Unidade Acadêmica de Engenharia Química \\ E-mail para contato: jocielys@yahoo.com.br/meiry@deq.ufcg.edu.br
}

\begin{abstract}
RESUMO - A contaminação por metais pesados, incentiva a busca de novas tecnologias de remediação. Diante de tal cenário, este trabalho visou contribuir no sentido de avaliar a preparação da peneira molecular SBA-15 e sua utilização como adsorvente no processo de separação óleo/água. Preparou-se a peneira molecular SBA-15 a partir de um gel com composição molar: 1.0 (Fonte de sílica): 0.017 P123 (Agente direcionador): $5.7 \mathrm{HCl}$ : 193 $\mathrm{H}_{2} \mathrm{O}$. As amostras foram caracterizadas por difração de raios $X(D R X)$, análise química por espectrometria de raios-X por energia dispersiva (EDX), Adsorção de Nitrogênio (Método de BET), testes de inchamento de Foster e testes de capacidade de adsorção em diversos solventes. Através do DRX, EDX e BET verificou-se a formação da peneira molecular mesoporosa. Os resultados dos testes em solventes orgânicos indicam que a SBA-15 apresenta capacidade de adsorção nos solventes orgânicos testados: gasolina $(1,788 \mathrm{~g} / \mathrm{g})$, querosene $(1,501 \mathrm{~g} / \mathrm{g})$ e diesel $(2,745 \mathrm{~g} / \mathrm{g})$.
\end{abstract}

\section{INTRODUÇÃO}

Um grande enfoque tem sido dado à preservação do meio ambiente, principalmente quando se trata de recursos naturais não renováveis. A fiscalização através dos órgãos competentes, com relação aos impactos ambientais e a preservação do meio ambiente têm estimulado as indústrias a se preocuparem com o tratamento dos seus efluentes (Fu e Wang, 2011).

Diariamente uma série de produtos orgânicos é descartada, provenientes de diversas fontes antrópicas como esgotos domésticos, efluentes industriais, atividades agropecuárias, produtos farmacêuticos, descartes de laboratórios, curtumes, refinarias de petróleo, entre outros. Diante disso, as pesquisas têm se intensificado com o intuito de melhorar o tratamento de efluentes industriais e assim, atenuar a poluição causada pelo lançamento de águas residuárias industriais em corpos d'água receptores. Com o desenvolvimento de novas tecnologias, os efluentes provenientes de indústrias vêm sofrendo constantes alterações em suas composições, através da inclusão de grande número de compostos químicos utilizados ou gerados na linha de processamento industrial (Ijagbemi et al., 2009; Cheremisinoff, 1995; Sabaté e Bayona, 2001).

Dentre as diversas formas de tratamento de efluentes poluídos por óleo destacam-se, precipitação química, troca iônica, adsorção, filtração por membranas, coagulação e floculação, 
flutuação e tratamento eletroquímico. Atualmente, a adsorção é reconhecida como método eficaz e econômico para o tratamento de águas oleosas. A adsorção é um processo que oferece flexibilidade no projeto e na operação, e em muitos casos após o tratamento, o efluente será um produto de alta qualidade. Dentre os materias promissores para utilização na remediação de corpos aquáticos, destacam-se as argilas, zeólitas e peneiras moleculares (Murillo et al., 2004; Sabaté e Bayona, 2001).

SBA-15 é uma nova classe de silicato mesoporoso que foi sintetizado inicialmente por Zhao et al. (1998). Este material mesoporoso com estrutura hexagonal altamente ordenada possui uma rede hexagonal de mesoporos de aproximadamente $6,0 \mathrm{~nm}$ de diâmetro e elevada área superficial. Além disto, a SBA-15 possui a parede dos poros relativamente mais espessa que aquelas das peneiras moleculares mesoporosas sintetizadas em meio básico (MCM-41 e MCM-48), resultando em uma maior estabilidade hidrotérmica (Zhao et al. 1998).

A SBA-15 é normalmente sintetizada em meio fortemente ácido, utilizando o tetraetilortosilicato (TEOS) como fonte de sílica tradicional e o copolímero tribloco Pluronic (P123Aldrich) como direcionador. A fonte de sílica pode ser selecionada através de critérios como disponibilidade, reatividade química e custo. A utilização do (TEOS) como fonte de sílica na síntese deste material onera o custo de preparação. Sob este aspecto é interessante testar novas fontes de sílica, tais como a cinza da casca de arroz, que apresenta em sua composição elevado teor de sílica e é encontrada no Brasil com muita facilidade além de ser uma matéria-prima barata e alternativa (Dey et al. 2013; Farshid et al. 2013; Renuka et al, 2013).

Portanto, este trabalho visa preparar a peneira molecular SBA-15 utilizando as cinzas da casca de arroz como fonte de sílica para avaliar seu potencial na capacidade de remoção de solventes orgânicos. As amostras foram caracterizadas por Difração de Raios X (DRX), Espectrômetro de Raios $\mathrm{X}$ por Energia Dispersiva (EDX e Adsorção física de $\mathrm{N}_{2}$ ), testes de inchamento de Foster e testes de capacidade de adsorção em diversos solventes.

\section{MATERIAIS E MÉTODOS}

\section{Ativação térmica da casca de arroz}

A casca de arroz foi submetida a um tratamento térmico em mufla a temperatura de $600{ }^{\circ} \mathrm{C}$ (patamares de $100{ }^{\circ} \mathrm{C}$ ) por um período de 4 horas. Ativação ácida da casca de arroz foi realizada com ácido clorídrico e as condições de ativação utilizadas foram: temperatura de $100{ }^{\circ} \mathrm{C}$, tempo de contato de 2 horas, 1 M concentração do ácido e uma razão mássica de casca de arroz/volume da solução $25 \mathrm{~g} / 500 \mathrm{~mL}$. Inicialmente, a solução aquosa foi preparada com $\mathrm{HCl}$ (1 M), a qual foi adicionada a amostra. $\mathrm{O}$ material foi seco por 12 horas a temperatura de $60^{\circ} \mathrm{C}$ (Lima et al. 2012).

\section{Síntese da peneira molecular SBA-15}

Para a síntese da peneira molecular SBA-15 foram utilizados os seguintes reagentes: Pluronic P123 (poli(óxido de etileno)-poli(óxido de propileno)-poli(óxido de etileno), $\mathrm{PEO}_{20} \mathrm{PPO}_{70} \mathrm{PEO}_{20}$ )) como direcionador, ácido clorídrico $37 \%$, água destilada, cinzas da casca de arroz como fonte de 


\section{9 a 22 de outubro de 2014 \\ Florianópolis/SC}

sílica, baseado no método hidrotérmico proposto por Zhao et al.(1998). Estes reagentes foram misturados em ordem para obter um hidrogel reativo com composição molar: 1,0 FONTE DE SÍLICA: 0,017 P123: 5,7 HCl: $193 \mathrm{H}_{2} \mathrm{O}$. A síntese foi realizada da seguinte forma: dissolveu-se o direcionador $\mathrm{P} 123$ em água destilada e $\mathrm{HCl}$, com agitação e aquecimento até $35^{\circ} \mathrm{C}$. Atingida a temperatura, adicionou-se a fonte de sílica. A mistura foi mantida sob agitação e aquecimento a $35^{\circ} \mathrm{C}$ por 24 horas $(\mathrm{pH}=1)$ para obter um gel homogêneo. Depois deste período o gel de síntese foi transferido para autoclave e acondicionado em estufa por 48 horas, previamente aquecida a $100^{\circ} \mathrm{C}$ $(8,9)$. Após esse processo o material obtido foi calcinado, em uma mufla, da temperatura ambiente até $550{ }^{\circ} \mathrm{C}$ com uma taxa de aquecimento de $10^{\circ} \mathrm{C} / \mathrm{min}$ permanecendo nesta temperatura por 24 horas.

\section{Caracterização}

Difração de raios $\mathrm{X}$ (DRX): Os difratogramas de raios-X foram realizados utilizando-se radiação de $\mathrm{K} \alpha$ de $\mathrm{Cu}$, com tensão acelerada $40 \mathrm{KV}$ e corrente de $40 \mathrm{~mA}$, com varredura de $0,5^{\circ}$ a $8^{\circ}$ para $2 \theta$, com velocidade de varredura de $2 \%$ min, realizados em um difratômetro XRD-6000 da Shimadzu.

Espectrometria de raios X por Energia Dispersiva (EDX): A composição química das amostras foi analisada em um espectrômetro EDX-700 Shimadzu.

Adsorção física de Nitrogênio (Método de BET): As características texturais dos catalisadores foram avaliadas a partir de isotermas de adsorção e dessorção de nitrogênio a $77 \mathrm{~K}$ obtidas através do equipamento ASAP 2020 da Micromeritics. Para isso $100 \mathrm{mg}$ de cada amostra foi previamente tratada nas as seguintes condições: Taxa de aquecimento: $10^{\circ} \mathrm{C} / \mathrm{min}$, Temperatura: $90^{\circ} \mathrm{C}$, Vácuo setpoint: 10 $\mu \mathrm{mHg}$, Tempo de vácuo: $60 \mathrm{~min}$. Fase de aquecimento: Taxa de aquecimento: $10^{\circ} \mathrm{C} / \mathrm{min} \mathrm{e}$ Temperatura de $350^{\circ} \mathrm{C}$.

Testes de Inchamento de Foster: Em uma proveta de $100 \mathrm{~mL}$ de capacidade, foi adicionado lentamente 1g SBA-15 a $50 \mathrm{~mL}$ do dispersante a ser estudado. Essa adição foi realizada lentamente, aguardando até a SBA-15 atingir umidade suficiente para a sedimentação. O sistema foi deixado em repouso por $24 \mathrm{~h}$ e então foi efetuada a leitura do inchamento sem agitação. Depois foi manualmente agitada, com bastão de vidro, durante 5 minutos, em seguida o sistema foi novamente deixado em repouso por mais 24 horas e então efetuada a leitura do inchamento com agitação. Os solventes orgânicos testados foram: diesel, gasolina e querosene.

Testes de Capacidade de Adsorção: O teste de capacidade de adsorção mede a afinidade que a SBA-15 tem com compostos orgânicos. Este teste é baseado na norma "Standard Methods of testing sorbent Performance of Absorbents" (ASTM F716-82, 1993). Este teste constou do seguinte procedimento: em um béquer de $600 \mathrm{~mL}$ colocou-se o solvente a ser testado até uma altura de $2 \mathrm{~cm}$. Em uma cesta (fabricada de tela de Aço Inoxidável com malha ABNT 200, abertura de 0,075 mm) colocou-se 1,00 g do material adsorvente (SBA-15) a ser testado. Esse conjunto foi pesado e colocado no recipiente com o solvente, onde permaneceu por 15 minutos. Após esse tempo, deixou-se fluir o excesso por 15 segundos e realizou-se uma nova pesagem. O teste foi realizado em triplicata visando 
obter resultados precisos. A quantidade de solvente adsorvida foi calculada usando a seguinte expressão:

$$
A d=\left(\frac{P_{1}-P_{2}}{P_{2}}\right) * 100
$$

Em que,

$\mathrm{P}_{1}$ é a massa do material após adsorção;

$\mathrm{P}_{2}$ é a massa do material adsorvente seco;

$\mathrm{A}_{\mathrm{d}}$ é a Capacidade de adsorção em gramas de solvente por gramas de SBA-15.

\section{RESULTADOS E DISCUSSÃO}

Os difratogramas de raios-X da peneira molecular SBA-15 estão apresentados na Figura 1.

a)

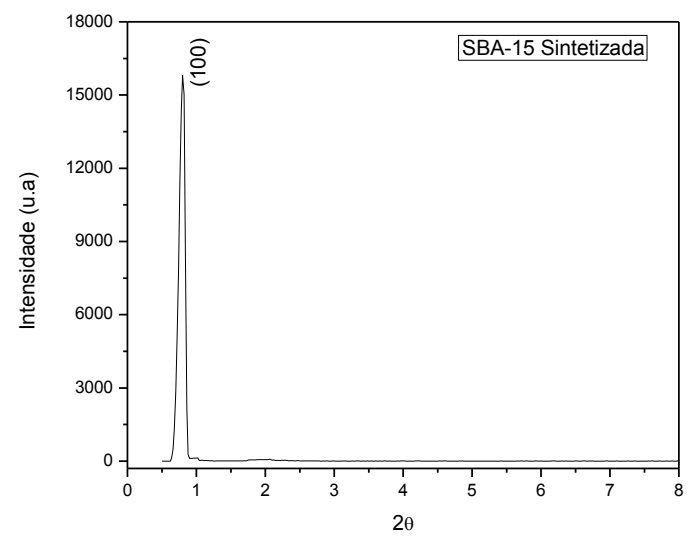

b)

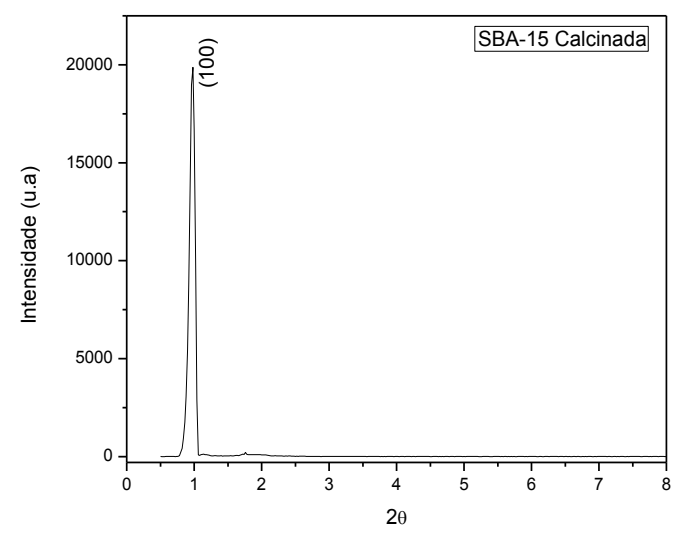

Figura 1 - Difratogramas de raios X da peneira molecular SBA-15: a) Sintetizada e b) Calcinada.

Os difratogramas de raios-X das amostras (SBA-15 sintetizada e calcinada) são apresentados na Figura 1a e 1b respectivamente. Através da análise dos difratogramas, é possível verificar que a estrutura hexagonal ordenada da peneira molecular mesoporosa SBA-15 está de acordo com a literatura (Zhao et al. 1998; Martinéz et al, 2003). Verifica-se a presença do pico principal de difração, característico desse material, referente ao plano cristalino, cujos índices de Miller são (1 0 0). Isto mostra que ao dissolver o direcionador Pluronic P123 em água e ácido clorídrico sob agitação e aquecimento $\left(35^{\circ} \mathrm{C}\right)$ são formadas as interações copolímero/solvente. Após a adição da fonte de sílica originam-se interações sílica/solvente para em seguida formar as interações sílica/copolímero. $\mathrm{O}$ gel obtido quando submetido ao processo de envelhecimento por $24 \mathrm{~h}$ sob agitação, pode resultar 
dependendo das condições da solução (temperatura, $\mathrm{pH}$ ) em uma fase siloxana pela condensação das espécies de sílica sobre as micelas direcionadoras do copolímero, e assim, o gel pode ser submetido a um tratamento hidrotérmico, para que aumente a espessura da parede de sílica, formando a peneira molecular SBA-15.

A Tabela 1 apresenta os resultados da análise química das amostras (SBA-15 sintetizada e calcinada).

Tabela 1 - Composição química das amostras (SBA-15 sintetizada e calcinada).

\begin{tabular}{|c|c|c|}
\hline Amostras & $\mathrm{SiO}_{2}(\%)$ & Outros (\%) \\
\hline SBA-15 (sintetizada) & 99,3 & 0,7 \\
\hline SBA-15 (calcinada) & 99,5 & 0,5 \\
\hline
\end{tabular}

De acordo com os resultados de composição química, verificou-se que as amostras em estudo apresentaram alto teor de sílica $\left(\mathrm{SiO}_{2}\right)$, visto que a peneira molecular possui em sua estrutura apenas sílica. As amostras apresentaram quantidades pequenas de impureza, isso diz respeito a contaminações presentes nas amostras, reagentes, e também a elementos que apresentam mesma energia de excitação (Zhao et al. 1998).

Na Figura 2 está exibida a isotermas de adsorção-dessorção de $\mathrm{N}_{2}$ a $-196{ }^{\circ} \mathrm{C}$ referentes à peneira molecular SBA-15 calcinada.

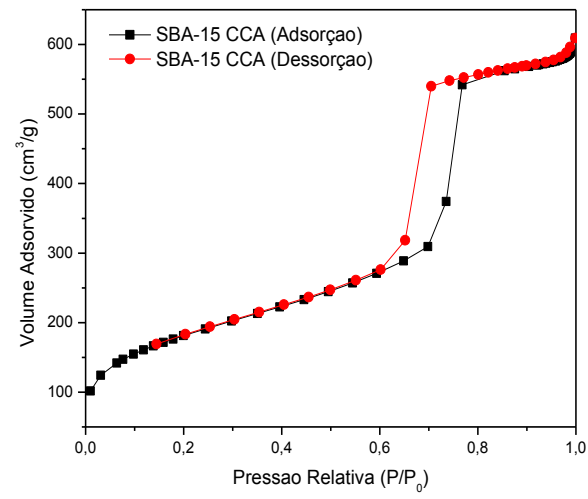

Figura 2 - Isotermas de adsorção-dessorção de $\mathrm{N}_{2}$ a $-196^{\circ} \mathrm{C}$ da peneira molecular SBA-15 calcinada.

De acordo com a IUPAC, As isotermas apresentadas são do tipo IV, característico de materiais mesoporosos. Verifica-se ainda o fenômeno de histerese tipo H1, caracterizada por dois ramos de isotermas quase verticais e paralelos durante uma extensa gama de valores do eixo das ordenadas (quantidade adsorvida) (Rodrigues et al. 2013, Zhao et al. 1998). Observa-se também que a isoterma de adsorção para a peneira molecular SBA-15 apresentou uma inflexão a uma pressão relativa no intervalo entre 0,6 - 0,8 indicativo de um material SBA-15 de boa qualidade com mesoporos uniformes. 
As propriedades texturais obtidas por adsorção de $\mathrm{N}_{2}$ da peneira molecular SBA-15 calcinada são apresentadas na Tabela 2.

Tabela 2 - Propriedades texturais da amostra da peneira molecular SBA-15 calcinada.

\begin{tabular}{|c|c|c|c|c|}
\hline Amostra & $\mathrm{S}_{\mathrm{BET}}\left(\mathrm{m}^{2} / \mathrm{g}\right)$ & $\mathrm{VP}_{\text {total }}\left(\mathrm{cm}^{3} / \mathrm{g}\right)$ & ${ }^{\mathrm{b}} \mathrm{D}_{\mathrm{p}}(\mathrm{nm})$ & $\mathrm{e}(\mathrm{nm})$ \\
\hline SBA-15 & 627 & 0,94 & 6,4 & 6,8 \\
\hline
\end{tabular}

Verifica-se que a área superficial específica, volume total de poros e diâmetro de poros da peneira molecular SBA-15 calcinada $\left(627 \mathrm{~m}^{2} / \mathrm{g}, 0,94 \mathrm{~cm}^{3} / \mathrm{g} \mathrm{e} 7,4 \mathrm{~nm}\right.$, respectivamente) são característicos de materiais mesoporosos sintetizados em condições similares (Zhao et al. 1998). Através da combinação das técnicas (DRX e BET) pode-se calcular a espessura da parede (Mendes et al. 2006) e o valor encontrado (6,8 nm) está de acordo com a literatura (Zhao et al. 1998)

Os resultados referentes às análises de capacidade de adsorção para a peneira molecular SBA15 são apresentados na Tabela 3.

Tabela 3 - Capacidade de adsorção em gramas de material adsorvido por grama da peneira molecular SBA-15.

\begin{tabular}{|c|l|}
\hline Solvente & SBA-15 \\
\hline Gasolina & $1,788(\mathrm{~g} / \mathrm{g})$ \\
\hline Querosene & $1,501(\mathrm{~g} / \mathrm{g})$ \\
\hline Diesel & $2,745(\mathrm{~g} / \mathrm{g})$ \\
\hline
\end{tabular}

Analisando os dados da Tabela 3 é possível verificar que os resultados de capacidade de adsorção da peneira molecular SBA-15 apresenta a seguinte relação: diesel > gasolina > querosene.

Os melhores resultados na adsorção com a peneira molecular SBA-15 foram obtidos utilizando o solvente orgânico diesel. Este fato indica provavelmente que existe uma melhor interação da peneira molecular SBA-15 com o solvente diesel. Essa interação está relacionada diretamente à composição química dos compostos (gasolina: $\mathrm{C}_{5}-\mathrm{C}_{10}$; querosene: $\mathrm{C}_{11}-\mathrm{C}_{12}$; diesel: $\mathrm{C}_{13}-\mathrm{C}_{17}$ ). Os resultados nos testes de adsorção podem também estar relacionados com a viscosidade dos solventes, tendo em vista que o óleo diesel é mais viscoso, uma vez que os resultados de viscosidade são: diesel - $8 \mathrm{mPa} . \mathrm{s}$ (600rpm) e 3,5 mPa.s (300rpm); querosene - $2 \mathrm{mPa} . \mathrm{s}$ (600rpm) e $1 \mathrm{mPa} . \mathrm{s}$ (300rpm); gasolina - 2 mPa.s (600rpm) e $1 \mathrm{mPa} . \mathrm{s}$ (300rpm).

O teste de Inchamento de Foster tem como finalidade avaliar o quanto a SBA-15 se dispersa e incha em compostos orgânicos. Este teste é baseado no Standard Test Method for Swell Index of Clay Mineral Component of Geosynthetic Clay Liners (ASTM D 5890-95).

Os resultados apresentados na Tabela 4 indicam a afinidade da peneira molecular SBA-15 em solventes orgânicos. 
Tabela 4 - Resultados dos testes de Inchamento de Foster da peneira molecular SBA-15.

\begin{tabular}{|c|c|c|}
\hline Solvente & SBA-15 sem agitação & SBA-15 com agitação \\
\hline Gasolina & 5 & 6 \\
\hline Querosene & 10 & 16 \\
\hline Diesel & 8 & 10 \\
\hline
\end{tabular}

Pode ser observado na Tabela 4, que a peneira molecular SBA-15 apresentou no processo sem agitação: baixo inchamento para o solvente gasolina, médio inchamento para diesel e alto inchamento para o querosene. E para o processo com agitação, é possível destacar: baixo inchamento para o solvente gasolina e alto inchamento para os solventes diesel e querosene.

\section{CONCLUSÃO}

Os padrões de difração de raios $\mathrm{X}$ apresentaram picos característicos da estrutura da peneira molecular SBA-15. As análises de adsorção física de $\mathrm{N}_{2}$ indicaram a obtenção de um material mesoporoso com elevada área superficial. Dessa forma, é possível sintetizar a peneira molecular SBA-15 empregando cinzas da casca de arroz como fonte de sílica. Os resultados obtidos para Inchamento de Foster mostraram melhores resultados no processo com agitação para o solvente diesel. Em relação ao teste de capacidade de adsorção em solventes orgânicos observou-se que a SBA-15 apresentou capacidade de adsorção nos solventes testados com uma tendência de: diesel > gasolina > querosene. Como conclusão geral, a peneira molecular SBA-15 utilizada como adsorvente na capacidade de adsorção de solventes orgânicos (gasolina, querosene e diesel) é bastante promissora para ser utilizada no processo de separação óleo/água.

\section{REFERÊNCIAS}

CHEREMISINOFF, P. N., Handbook of Water and Wastewater Treatment Technology, Marcel Dekker Inc., New York, 1995.

DEY, K. P.; GHOSH, S.; MNASKAR, K. M. A facile synthesis of ZSM-11 zeolite particles using rice husk ash as silica source, Materials Letters, v.87, p. 87-89, 2012.

FARSHID, G.; HABIBOLLAH, Y.; ZAHRA, M.; MEHMET, S. C. E.; ALI, A. G.; MANSOOR, A. Preparation and characterization of highly pure silica from sedge as agricultural waste and its utilization in the synthesis of mesoporous silica MCM-41. Journal of the Taiwan Institute of Chemical Engineers. In press, 2013.

FU, F., WANG, G., Removal of heavy metal íons from wastewaters: A review. Journal of Environmental Management. v. 92, p. 407-418, 2011. 
IJAGBEMI, C. O.; BAEK, M.; KIM, D.; Montmorillnite surface properties and sorption characteristics for heavy metal removal from aqueous solutions. Journal of Hazardous Materials, v. 166, p. 538-546, 2009.

LIMA, L. A.; RODRIGUES, M. G. F. Preparação da peneira molecular MCM-41 usando a cinza da casca de arroz como fonte de sílica. In: IX Encontro Norte Nordeste Centro Oeste de Catálise, Maceió, 2012.

MARTÍNEZ, A.; LÓPEZ, C.; MÁRQUEZ, F.; DÍAZ, I. Fischer-Tropsch synthesis of hydrocarbons over mesoporous Co/SBA-15 catalysts: the influence of metal loading, cobalt precursor, and promoters. Journal of Catalysis, v.220, p.486-499, 2003.

MENDES, F M T; PEREZ, C A C; NORONHA, F B; SOUZA, C D D; CESAR, D V; FREUND, H J; SCHMAL, M. Fischer-Tropsch Synthesis on Anchored Co/Nb2O5/A12O3 Catalysts: The Nature of the Surface and the Effect on Chain Growth. Journal of Physical Chemistry, vol. 110, p. 9155-9163, 2006.

MURILlO, R.; GARCIA, T.; AYLÓN, E.; CALLÉN, M.S.; NAVARRO, M.V.; LÓPEZ, J. M.; MASTRAL, A.M. Adsorption of phenanthrene on activated carbons: Breakthrough curve modeling. Carbon, v. 42, 2009 -2017, 2004.

RENUKA, N. K.; PRAVEEN, A.K.; ANAS, K. Influence of CTAB molar ratio in tuning the texture of rice husk silica into MCM 41 and SBA-16. Materials Letters. v.109, p.70-73, 2013.

RODRIGUES, J. J.; FERNANDES, F.A.N.; RODRIGUES, M. G. F. Study of Co/SBA-15 catalysts prepared by microwave and conventional heating methods and application in Fischer-Tropsch synthesis. Appl. Catal. A. v.468, p.32-37, 2013.

SABATÉ, J.; BAYONA, J. M. Photolysis of PAHs in aqueous phase by UV irradiation Chemosphere, v. 44, p. 119 -124, 2001.

STANDARD METHODS OF TESTING SORBENT PERFORMANCE OF ADSORBENTS DESIGNATION; ASTM F716 -82 (REAPPROVED 1993).

\section{STANDARD TEST METHOD FOR SWELL INDEXO F CLAY MINERAL COMPONENT OF GEOSYNTHETIC CLAY LINERS - ASTM D 5890-95.}

ZHAO, E., FENG, J., HUO, Q, FENG, J..G.H., CHMELKA, B.F., STUCKY, G.D. Nonionic triblock copolymer and oligameric surfactant syntheses of highly ordered hydrothermally stable, mesoporous sílica structures. J. Am. Chem. Soc. v.120, p. 6024-6036, 1998. 